\begin{tabular}{|c|l|}
\hline Title & Type II behavior in wurtzite InP/nA s/nP core multishell nanowires \\
\hline Author(s) & Pal, B.; Goto, K.; Ikezawa, M.; Masumoto, Y.; Mohan, P.; Motohisa, J.; Fukui, T. \\
\hline Citation & $\begin{array}{l}\text { A pplied Physics Letters, 93/7), 073105 } \\
\text { https://doi.org/_0.1063/2966343 }\end{array}$ \\
\hline Issue Date & 2008_08 \\
\hline Doc URL & http://hdl.handle.net/2115/35016 \\
\hline Rights & $\begin{array}{l}\text { Copyright 2008_A merican Institute of Physics. This article may be downloaded for personal use only. A ny other use } \\
\text { requires prior permission of the author and the A merican Institute of Physics. }\end{array}$ \\
\hline Type & article \\
\hline File Information & A pplPhysLett_93_073105.pdf \\
\hline
\end{tabular}

Instructions for use 


\title{
Type-II behavior in wurtzite InP/InAs/InP core-multishell nanowires
}

\author{
B. Pal, ${ }^{1, a)}$ K. Goto, ${ }^{1}$ M. Ikezawa, ${ }^{1}$ Y. Masumoto, ${ }^{1}$ P. Mohan, ${ }^{2}$ J. Motohisa, ${ }^{2}$ and T. Fukui ${ }^{2}$ \\ ${ }^{1}$ Institute of Physics, University of Tsukuba, Tsukuba 305-8571, Japan \\ ${ }^{2}$ Research Center for Integrated Quantum Electronics, Hokkaido University, Sapporo 060-8628, Japan
}

(Received 13 June 2008; accepted 13 July 2008; published online 20 August 2008)

\begin{abstract}
We study optical transitions from a periodic array of InP/InAs/InP core-multishell nanowires (CMNs) having a wurtzite crystal structure by using photoluminescence (PL) and PL excitation (PLE) spectroscopy. Observing a large Stokes shift between PL and PLE spectra, a blueshift of the PL peak with a cube-root dependence on the excitation power and a slow and nonexponential decay of PL with an effective decay time of 16 ns suggest a type-II band alignment. Band-offset calculation based on the "model-solid theory" of Van de Walle [Phys. Rev. B 39, 1871 (1989)] supports type-II band lineup if the InAs layer in the wurtzite CMNs is assumed to sustain compressive strain in all directions. (0) 2008 American Institute of Physics.
\end{abstract}

[DOI: $10.1063 / 1.2966343]$

Nanometer-scale semiconductor heterostructures such as quantum dots, quantum wires, and quantum wells (QWs) have been interesting research targets due to their unique size-dependent electronic and optical properties associated with the lower dimensionality and quantum confinement effects. There have been revived interests in semiconductor nanowires (NWs) due to recent success in growth and fabrication of a regular array of core-shell and core-multishell NWs (CMNs). ${ }^{1,2}$ Semiconductor NWs can be used as building blocks of sophisticated nanoscale electronic and photonic devices. ${ }^{3}$ Much effort has been devoted so far to the growth and fabrication of CMNs and the periodic arrays of such structures. ${ }^{1,2}$ However, very little is known about the electronic structure and optical properties of this new class of technologically important structures.

In this letter we report optical studies on the periodic array of InP/InAs/InP CMNs. Both InP and InAs have a wurtzite crystal structure in the NW form, ${ }^{4-6}$ although bulk InP and InAs crystallize to a zinc-blende structure. Moreover, in our CMN sample a thin InAs layer is surrounded by thick InP layers from all sides and the InP layers act like a mold. As a first approximation, we may consider that the InAs lattice experiences a three-dimensional compressive strain and attains the size of the InP lattice in all directions unlike InAs/InP QWs, where the InAs lattice is compressed in the crystal growth plane and is elongated in the growth direction. There have been a few studies on ultrathin InAs/ InP QWs having zinc-blende structure. ${ }^{7}$ These experimental results and theoretical calculations using an envelopefunction scheme with effective-mass approximation and empirical tight-binding model ${ }^{8}$ seem to show type-I direct transitions in zinc-blende InAs/InP QWs, although some calculations also predict type-II behavior. ${ }^{9}$ In contrast, the electronic structure and optical properties of wurtzite $\mathrm{InP}$ and InAs are almost unknown. ${ }^{5,10}$ Due to the differences in crystal structure and strain, the insights gathered from the studies on zinc-blende InAs/InP QWs cannot be applied directly to wurtzite InP/InAs/InP CMNs.

\footnotetext{
${ }^{a)}$ Present address: Indian Institute of Science Education and ResearchKolkata, Kolkata 700 106, India. Electronic mail: bipulpal@gmail.com.
}

We study the InP/InAs/InP CMNs using time-resolved (TR) and spectrally resolved (SR) photoluminescence (PL) and PL excitation (PLE) measurements. A large Stokes shift between PL and PLE spectra was observed, which along with the absence of strong PLE peak suggests type-II radiative recombination. With increasing excitation power $(P)$ the PL peaks show a blueshift with a cube-root dependence on $P$, confirming type-II radiative transition. TR-PL measurements using time-correlated single photon counting (TCSPC) technique show a slow and nonexponential decay of PL with an effective decay time of $16 \mathrm{~ns}$, as expected for type-II optical transitions. Band-offset calculation based on the "model-solid theory" of Van de Walle ${ }^{11}$ for the wurtzite InP/ InAs/InP heterostructure assuming three-dimensional compressive strain supports type-II band lineup.

Our sample is the periodic array of vertically oriented and highly uniform InP/InAs/InP CMNs grown by using selective area metal organic vapor phase epitaxy. Details of the sample growth procedure and scanning electron microscopy images of the grown structure are given in Ref. 2. Transmission electron microscopy studies revealed that InP and InAs have a wurtzite crystal structure in these CMNs. ${ }^{4,5}$ Schematics of the horizontal and vertical cross sections of a CMN is shown in Fig. 1(a). A tunable cw Ti:sapphire laser was used in the SR-PL (spectral resolution of $\sim 0.02 \mathrm{meV}$ ) and PLE measurements performed at $2 \mathrm{~K}$. The standard TCSPC

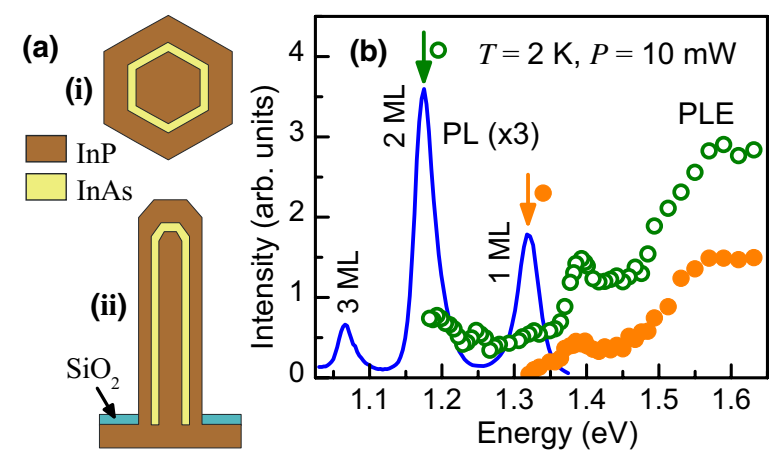

FIG. 1. (Color online) (a) Schematics of the (i) horizontal and (ii) vertical cross sections of the CMN. (b) PL (solid line) and PLE spectra for detection (indicated by arrows) at $1 \mathrm{ML}$ (filled circles) and $2 \mathrm{ML}$ (open circles) PL peaks. 


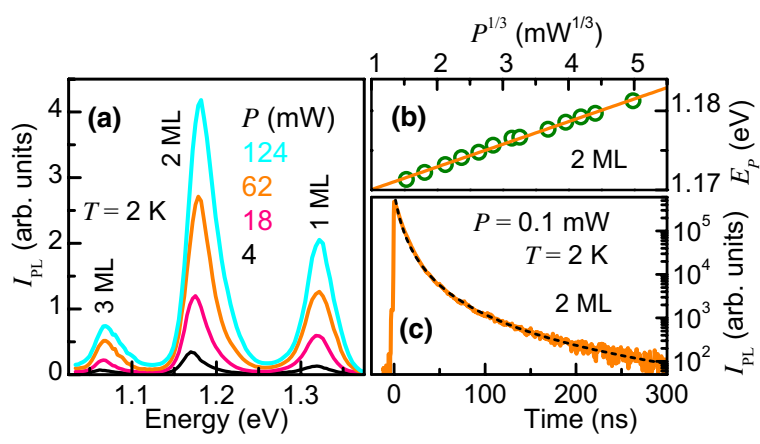

FIG. 2. (Color online) (a) PL spectra at a few excitation powers $P$. (b) PL peak energy position $E_{P}$ as a function of $P^{1 / 3}$. (c) TR-PL data (solid line) along with a fit (dashed line) as discussed in the text. ( $I_{\mathrm{PL}}=$ PL intensity).

technique $^{12}$ (time resolution of $\sim 1 \mathrm{~ns}$ ) was used for TR-PL measurements. Our PL measurements were performed for excitation at $1.38 \mathrm{eV}$, below the wurtzite InP bandgap of 1.5 $\mathrm{eV},{ }^{13}$ to avoid the complication of carrier relaxation in the InP barrier. Focused laser spot diameter in our experiments is $\sim 150 \mu \mathrm{m}$, covering $\sim 10^{5}$ CMNs. We estimate that for $1 \mathrm{~mW} \mathrm{cw}$ excitation, the steady-state carrier density is less than three electron-hole pairs per CMN on average.

Low-temperature PL and PLE spectra of the sample measured for $P=10 \mathrm{~mW}$ are shown in Fig. 1(b). Multiple peaks in PL spectra arise due to one monolayer variation in the InAs layer thickness. ${ }^{15}$ We designate the three PL peaks as 1-3 ML peaks based on a calculation of the ground-state transition energy in a strained InAs/InP QWs. ${ }^{2}$ The shoulderlike structures at 1.25 and $1.39 \mathrm{eV}$ in PLE spectra may be assigned to the absorption for 2 and $1 \mathrm{ML}$ regions, respectively. A rise in the PLE signal at $\sim 1.5 \mathrm{eV}$ is related to the absorption in the InP layer. ${ }^{13}$ We observe a Stokes shift of 70 meV between PL and PLE spectra. The full width at half maximum (FWHM) of PL peaks is $30 \mathrm{meV}$. Broad PL peaks may be caused by the inhomogeneous broadening arising from short-range monolayer fluctuations and intermixing of As and $\mathrm{P}$ at the interface. However, the Stokes shift is too large to be explained by the inhomogeneous broadening only. It is known that for QWs the Stokes shift for the inhomogeneously broadened exciton PL band almost universally becomes 0.6 times the FWHM of the excitonic absorption band. This universality is based on a large number of experimental observations and is also supported by theoretical calculations. ${ }^{16}$ In our case the Stokes shift is more than twice the PL linewidth. Relaxation in an inhomogeneously broadened band cannot explain such a large Stokes shift. We consider that the large Stokes shift observed here is due to a type-II transition ${ }^{17}$ because the absorption takes place at a higher energy than the PL in a type-II system. Also, the absorption transitions appear as shoulders rather than strong peaks in PLE spectra. The absence of strong PLE peaks [Fig. 1(b)] is consistent with the type-II behavior.

It has been shown in the literature by several authors ${ }^{18}$ that for a type-II optical transition a blueshift of the PL peak for increasing excitation power $P$ is observed with a cuberoot dependence on $P$. In the type-II system the $P^{1 / 3}$ dependence of the transition energy arises from the band bending effect due to spatially separated electron and holes. ${ }^{18,19} \mathrm{PL}$ spectra from our sample for a few values of $P$ are plotted in Fig. 2(a). Blueshift of all the peaks with increasing $P$ is evident. Peak energy position $E_{P}$ as a function of $P^{1 / 3}$ for the
2 ML peak fits very well to a straight line in Fig. 2(b). Identical behavior was observed in other peaks. Note that the blueshift of the PL peak linear with $P$ due to state filling effect (Pauli exclusion principle) is predicted theoretically ${ }^{20}$ and observed experimentally ${ }^{21}$ in type-I QWs. We find in our experiments that the PL intensity $\left(I_{\mathrm{PL}}\right)$ increases linearly with $P$ without any saturation in the range of $P$ used. This shows that the recombination is excitonic in nature, and the excitation is in the low power regime where the state filling effect is negligible. Therefore, we consider that the $P^{1 / 3}$ dependence of the PL blueshift observed in Fig. 2(b) arises due to the type-II nature of our wurtzite InP/InAs/InP CMN sample.

For type-II transitions PL decay time is known to be long (several tens of nanoseconds) due to reduced overlap of the electron-hole wave functions. ${ }^{22}$ Decay of PL signal at the 2 ML peak recorded using a standard TCSPC (Ref. 12) setup is shown in the semilogarithmic plot in Fig. 2(c). We observe a slow and nonexponential decay. Identical behavior was observed in other peaks. This indicates type-II transition. ${ }^{23} \mathrm{We}$ note that nonradiative recombination is negligible in our sample at low temperatures because temperature dependence of PL shows that integrated PL intensity and PL decay rate are nearly independent of temperature up to $50 \mathrm{~K}$. We attribute the nonexponential decay of PL in Fig. 2(c) to a distribution of decay rates arising from inhomogeneities at the interface due to monolayer fluctuations and intermixing of As and P. Nonexponential decay resulting from a distribution of decay rates is often fitted ${ }^{25}$ with the function $I_{\mathrm{PL}}(t)=I_{0}(1$ $+2 \Gamma t)^{-\beta}$ if nonradiative decay can be neglected, as it is in our case. Here, $I_{0}$ is the PL intensity at $t=0, \Gamma$ is an effective decay rate, and the exponent $\beta$ depends on decay mechanism, distribution of decay rates, etc. We fit the TR-PL data in Fig. 2(c) using the above function. Best fit to our data yields $\beta=2.5 \pm 0.02$ and $\Gamma=0.06 \pm 0.006 \mathrm{~ns}^{-1}$, giving an effective PL decay time of about $16 \mathrm{~ns}$.

Calculation of electronic energy levels for wurtzite InP/ InAs/InP CMNs having three-dimensional strain distribution with cross section in hexagonal symmetry and with translational symmetry along the NW axis is a complex task and is not available in the literature. For simplicity, we take the following approaches to calculate the conduction and valance band offsets based on the model-solid theory of Van de Walle: ${ }^{11}$ In the first approach, we treat the InAs layer as a strained QW on the InP (110) surface. ${ }^{2}$ In the second approach, the strain is calculated assuming that the $\mathrm{CMN}$ was radially symmetric and taking the lattice constant in the axial direction as that of InP. In both the approaches calculation parameters are taken as that of zinc-blende InAs and InP, and they lead to a type-I band alignment because of biaxial strain. However, InAs and InP have a wurtzite crystal structure in our sample, and a thin InAs shell is completely buried between thick InP layers [Fig. 1(a)], which will force the InAs lattice constant in the radial direction to be that of InP. Also the epitaxial growth of the sample makes the InAs lattice constant in the axial direction the same as that of InP. We may assume that the InAs layer is under hydrostatic pressure, which results in compressive strains in all three directions. ${ }^{26-28}$ We take the $c$-axis lattice constant of wurtzite InAs (Ref. 2) (InP) (Ref. 4) to be $c=6.9(6.6) \AA$ and use $c / a=1.633$ ( $a$ is the lattice constant in the plane perpendicular to the $c$-axis). ${ }^{29}$ Then using the formalism and parameters from Ref. 11, we obtain the type-II band alignment with the 
conduction (valance) band of InAs lying 0.16 (0.32) $\mathrm{eV}$ above the conduction (valance) band of InP, which is in agreement with our experimental results.

In conclusion, we study optical properties of the periodic array of InP/InAs/InP CMNs by TR- and SR-PL and PLE measurements. Our study suggests a type-II optical transition arising due to wurtzite crystal structure and triaxial compressive strain in these novel nanostructures.

The work is partially supported by "Grant-in-Aid for Scientific Research" under Grant Nos. 20244044 and 17-5056 from the MEXT of Japan.

${ }^{1}$ L. J. Lauhon, M. S. Gudiksen, D. Wang, and C. M. Lieber, Nature (London) 420, 57 (2002); N. Sköld, L. S. Karlsson, M. W. Larsson, M.-E. Pistol, W. Seifert, J. Trägårdh, and L. Samuelson, Nano Lett. 5, 1943 (2005); M. Liu, X. Li, H. Imrane, Y. Chen, T. Goodrich, Z. Cai, K. S. Ziemer, and J. Y. Huang, Appl. Phys. Lett. 90, 152501 (2007).

${ }^{2}$ P. Mohan, J. Motohisa, and T. Fukui, Appl. Phys. Lett. 88, 133105 (2006). ${ }^{3}$ See, e.g., Y. Cui and C. M. Lieber, Science 291, 851 (2001); X. Duan, Y. Huang, Y. Cui, J. Wang, and C. M. Lieber, Nature (London) 409, 66 (2001).

${ }^{4}$ P. Mohan, J. Motohisa, and T. Fukui, Nanotechnology 16, 2903 (2005).

${ }^{5}$ P. Mohan, J. Motohisa, and T. Fukui, Appl. Phys. Lett. 88, 013110 (2006).

${ }^{6}$ Z. Zanolli, M.-E. Pistol, L. E. Fröberg, and L. Samuelson, J. Phys.: Condens. Matter 19, 295219 (2007).

${ }^{7}$ See, e.g., R. Houdré, J. F. Carlin, A. Rudra, J. Ling, and M. Ilegems, Superlattices Microstruct. 13, 67 (1993); A.-M. Vasson, A. Vasson, J. Leymarie, P. Disseix, P. Boring, and B. Gil, Semicond. Sci. Technol. 8, 303 (1993); R. Leonelli, C. A. Tran, J. L. Brebner, J. T. Graham, R. Tabti, R. A. Masut, and S. Charbonneau, Phys. Rev. B 48, 11135 (1993); P. Paki, R. Leonelli, L. Isnard, and R. A. Masut, Appl. Phys. Lett. 74, 1445 (1999).

${ }^{8}$ N. Shtinkov, P. Desjardins, and R. A. Masut, Phys. Rev. B 66, 195303 (2002).

${ }^{9}$ V. Albe and L. J. Lewis, Physica B 301, 233 (2001).

${ }^{10}$ M. Murayama and T. Nakayama, Phys. Rev. B 49, 4710 (1994); M. Mattila, T. Hakkarainen, M. Mulot, and H. Lipsanen, Nanotechnology 17, 1580 (2006).

${ }^{11}$ C. G. Van de Walle, Phys. Rev. B 39, 1871 (1989).

${ }^{12}$ D. V. O'Conner and D. Phillips, Time-Correlated Single Photon Counting (Academic, New York, 1984).
${ }^{13}$ The bandgap of wurtzite $\mathrm{InP}$ is larger by $\sim 80 \mathrm{meV}$ compared to that of zinc-blende InP (Refs. 6, 10, and 14).

${ }^{14}$ Y. Kobayashi, M. Fukui, J. Motohisa, and T. Fukui, Physica E (Amsterdam) 40, 2204 (2008).

${ }^{15}$ Multipeak PL spectra due to monolayer fluctuations are commonly observed for thin InAs/InP QWs (Ref. 7).

${ }^{16}$ F. Yang, M. Wilkinson, E. J. Austin, and K. P. O’Donnell, Phys. Rev. Lett. 70, 323 (1993)

${ }^{17}$ T. T. Chen, C. H. Chen, W. Z. Cheng, W. S. Su, M. H. Ya, Y. F. Chen, P. W. Liu, and H. H. Lin, J. Appl. Phys. 93, 9655 (2003).

${ }^{18}$ X. D. Luo, C. Y. Hu, Z. Y. Xu, H. L. Luo, Y. Q. Wang, J. N. Wang, and W. K. Ge, Appl. Phys. Lett. 81, 3795 (2002); J. Hellara, K. Borgi, H. Maaref, V. Souliere, and Y. Monteil, Mater. Sci. Eng., C 21, 231 (2002); T. T. Chen, C. L. Cheng, Y. F. Chen, F. Y. Chang, H. H. Lin, C.-T. Wu, and C.-H. Chen, Phys. Rev. B 75, 033310 (2007).

${ }^{19}$ N. N. Ledentsov, J. Böhrer, M. Beer, F. Heinrichsdorff, M. Grundmann, D. Bimberg, S. V. Ivanov, B. Ya. Meltser, S. V. Shaposhnikov, I. N Yassievich, N. N. Faleev, P. S. Kop'ev, and Zh. I. Alferov, Phys. Rev. B 52, 14058 (1995)

${ }^{20}$ S. Schmitt-Rink, D. S. Chemla, and D. A. B. Miller, Phys. Rev. B 32, 6601 (1985).

${ }^{21}$ See, e.g., Y. Masumoto, S. Shionoya, and H. Okamoto, Opt. Commun. 53, 385 (1985).

${ }^{22}$ C.-K. Sun, G. Wang, J. E. Bowers, B. Brar, H.-R. Blank, H. Kroemer, and M. H. Pilkuhn, Appl. Phys. Lett. 68, 1543 (1996).

${ }^{23}$ Long decay time in Fig. 2(c) cannot come from type-I transitions. Recently we have studied zinc-blende 2 ML type-I InAs/InP QWs that show a PL decay time of 1-2 ns (Ref. 24).

${ }^{24}$ S. Tomimoto, A. Kurokawa, Y. Sakuma, T. Usuki, and Y. Masumoto, Phys. Rev. B 76, 205317 (2007).

${ }^{25}$ M. V. Klein, M. D. Sturge, and E. Cohen, Phys. Rev. B 25, 4331 (1982); F. Minami, K. Hirata, K. Era, T. Yao, and Y. Masumoto, ibid. 36, 2875 (1987); E. Finkman, M. D. Sturge, M.-H. Meynadier, R. E. Nahory, M. C. Tamargo, D. M. Hwang, and C. C. Chang, J. Lumin. 39, 57 (1987).

${ }^{26} \mathrm{We}$ assume no deformation in the lattice of the thick InP layers.

${ }^{27}$ Dependence of bandgap and energy levels on hydrostatic pressure for InAs is discussed in the literature. See, e.g., C. Kristukat, A. R. Goñi, K. Pötschke, D. Bimberg, and C. Thomsen, Phys. Status Solidi B 244, 53 (2007).

${ }^{28}$ The strain distribution may be highly nonuniform due to the corners of the hexagonal NWs.

${ }^{29}$ M. W. Larsson, J. B. Wagner, M. Wallin, P. Håkansson, L. E. Fröberg, L. Samuelson, and L. R. Wallenberg, Nanotechnology 18, 015504 (2007). 